This item was submitted to Loughborough's Research Repository by the author.

Items in Figshare are protected by copyright, with all rights reserved, unless otherwise indicated.

\title{
Generic twistless bifurcations
}

PLEASE CITE THE PUBLISHED VERSION

\section{LICENCE}

CC BY-NC-ND 4.0

\section{REPOSITORY RECORD}

Dullin, Holger R., J.D. Meiss, and D.G. Sterling. 2019. "Generic Twistless Bifurcations". figshare. https://hdl.handle.net/2134/840. 


\title{
Generic Twistless Bifurcations
}

\author{
H. R. Dullin ${ }^{1,2}$, J. D. Meiss ${ }^{1}$, D. Sterling ${ }^{2}$ * \\ ${ }^{1}$ Department of Applied Mathematics, \\ University of Colorado, Boulder, CO 80309-0526, USA \\ ${ }^{2}$ Department of Mathematical Sciences, \\ University of Loughborough, LE11 3TU, UK
}

H.R.Dullin@lboro.ac.uk, jdm@Colorado.EDU, sterling@Colorado.EDU

October 29, 1999

\begin{abstract}
We show that in the neighborhood of the tripling bifurcation of a periodic orbit of a Hamiltonian flow or of a fixed point of an areapreserving map, there is generically a bifurcation that creates a "twistless" torus. At this bifurcation, the twist, which is the derivative of the rotation number with respect to the action, vanishes. The twistless torus moves outward after it is created and eventually collides with the saddle-center bifurcation that creates the period three orbits. The existence of the twistless bifurcation is responsible for the breakdown of the nondegeneracy condition required in the proof of the KAM theorem for flows or the Moser twist theorem for maps. When the twistless torus has a rational rotation number, there are typically reconnection bifurcations of periodic orbits with that rotation number.
\end{abstract}

Keywords: Twist Maps; Hamiltonian Systems; Tripling Bifurcation; Reconnection bifurcation; Normal Forms; Abelian Integrals

*HRD was supported in part by DFG grant Du 302/2. JDM was supported in part by NSF grants DMS-9623216 and DMS-9971760. DS was supported by an NSF Graduate Traineeship under DMS-9256335 


\section{Introduction}

The dynamics in the neighborhood of an elliptic periodic orbit of a two-degree of freedom Hamiltonian flow, or equivalently, an elliptic fixed point of an areapreserving map, can be elucidated by consideration of their formal normal forms. When the rotation number, $\omega$, of the elliptic orbit is irrational, the normal form is called the Birkhoff normal form. Let $J$ denote the transverse action (the "symplectic radius") and $\theta$ be its conjugate angle. The Birkhoff normal form for the Hamiltonian is

$$
H(J)=\omega J+\frac{1}{2} \tau_{0} J^{2}+\frac{1}{6} \tau_{1} J^{3}+\ldots
$$

For an area-preserving map with an elliptic fixed point, the Birkhoff normal form is

$$
\begin{aligned}
J & \mapsto J \\
\theta & \mapsto \theta+2 \pi \Omega(J),
\end{aligned}
$$

where the rotation number is

$$
\Omega(J)=\omega+\tau_{0} J+\frac{1}{2} \tau_{1} J^{2}+\ldots
$$

This map is also the time $2 \pi$ map of the Hamiltonian flow. We define the "twist" to be the derivative of the transverse rotation number with respect to the action:

$$
\tau(J) \equiv \frac{d \Omega}{d J}=\tau_{0}+\tau_{1} J+\ldots
$$

In general, the Birkhoff series does not converge and so strictly speaking the function $\Omega$ does not exist unless the system is integrable. The truncated Birkhoff normal form gives an integrable approximation with a rotation number $\Omega(J)$ that approximates the rotation numbers of the Cantor set of KAM tori near the fixed point of the full system. The actual dynamics of the generic, non-integrable system includes a chain of $n$ islands located near each radius where $\Omega$ (of the truncated normal form) is $m / n$, a rational number. This chain is constructed from an elliptic and hyperbolic pair of periodic orbits of period $n$. These orbits are born in an $n$-tupling bifurcation that occurs when $\omega$ passes through the rational value $m / n$. 
The character of the bifurcation as $\omega$ passes through $m / n$ when $\tau_{0} \neq 0$ depends upon $n[3,10,12]$. When $n>4$, the elliptic orbit remains stable, and the bifurcation gives rise to a chain of $n$ islands. As we recall in the Appendix, for $n=4$ the origin is unstable if the resonant term is large enough. When $n=3$, the resonant term generically dominates the twist term near the origin, and the origin is unstable at the bifurcation point.

When the rotation number $\Omega$ of the normal form is a monotone function of $J$, or equivalently, the twist does not vanish, the map is called a "monotone twist map." The nonvanishing of the twist also corresponds to the isoenergetic nondegeneracy condition required for KAM theory [2], in the proof of the existence of invariant tori. A related, but slightly different twist condition is given by $\partial \Theta^{\prime} / \partial J \neq 0$, which is defined for the full non-integrable map of the cylinder. For this case, Aubry-Mather theory applies [9] and implies in particular that there is a pair of rotational $m / n$ periodic orbits for each rational in the range of $\Omega$.

When the twist vanishes, the dynamics can be much more complicated. For example, "reconnection bifurcations," occur near an extremal point of $\Omega$ $[8,7]$, and the renormalization operator for the destruction of invariant circles gives a distinct universality class for circles that cross the zero twist line as opposed to those which do not [4]. Recently in [15] it has been shown that the "meandering curves" that appear when a twistless curve passes through a rational rotation number are stable under small perturbations. An extension of standard KAM theory [5] shows that in two parameter families of areapreserving maps a diophantine twistless curve persists.

While it appears that $\tau_{0}$ should generically be nonzero, and so the twist is nonvanishing at least in some neighborhood of the origin, we will show in this paper that this is not true whenever the rotation number $\omega$ passes through $1 / 3$. This answers the genericity question raised in [15].

To show that the twist generically vanishes, we begin with the normal form in the neighborhood of a tripling bifurcation. Generally, when the rotation number of the elliptic periodic orbit is rational, there are resonant terms that cannot be transformed away. If we keep only the first such resonant term, the Hamiltonian becomes (see e.g. $[3,10]$ )

$$
\tilde{H}(I, \theta, t)=\omega I+A I^{2}+\cdots+B I^{n / 2} \cos (n \theta-m t)+\ldots .
$$

We now use the variable $I$ to denote the "action" for this system; it is not a true action variable. Here the system is at resonance when $\omega=m / n$. The 
time dependence in $\tilde{H}$ can be eliminated by a transformation to rotating coordinates, defining $\phi=\theta-m t / n$. This gives the new Hamiltonian $H=$ $\tilde{H}-m I / n$ :

$$
H(I, \phi)=\varepsilon I+A I^{2}+\cdots+B I^{n / 2} \cos (n \phi)+\ldots,
$$

where $\varepsilon=\omega-m / n$ measures the frequency difference from the resonant case. The time $2 \pi$ map of $\tilde{H}$ is an area preserving map which has $H$ as a conserved quantity. In the rotating frame a period $n$ orbit of the stroboscopic map of $\tilde{H}$ becomes a family of $n$ fixed points of the stoboscopic map of $H$.

When the resonant coefficient $B$ vanishes, then the coordinate $I$ is the true action, $J$, and the twist for Eq. (4) is $\tau=2 A+O(J)$, as we can see by comparison with Eq. (1). However, when $B \neq 0$, the action is modified and so is the twist. Moreover when $n=3$, the resonant term is of lower order than the first twist term, and even the $O\left(J^{0}\right)$ terms in the twist must be corrected. We will compute the twist for this system in $\S 2$.

We also consider the resonant normal form for an area-preserving map, $(p, q) \mapsto\left(p^{\prime}, q^{\prime}\right)$ where $(p, q)$ are cartesian canonical coordinates. These coordinates are related to the action-angle variables by the complex transformation.

$$
z \equiv \sqrt{2 I} e^{i \theta}=p+i q
$$

The resonant normal form, which is most easily expressed as a map on $z$ and in terms of the multiplier of the fixed point

$$
\lambda \equiv e^{2 \pi i \omega}
$$

is

$$
z^{\prime}=\lambda\left(z+i \alpha z^{2} \bar{z}+\cdots+\beta \bar{z}^{n-1}+\ldots\right)
$$

The omitted terms, as we will see below, include terms that are required for the map to be area-preserving. By comparison with Eq. (2), the twist when $\beta=0$ is $\tau=\alpha / \pi+O(J)$. The resonant terms, however, will modify the twist; indeed when $n=3$, the resonant terms are of lower order than the first twist term, and so even the $O\left(J^{0}\right)$ terms in $\tau$ should be changed.

We will show that whenever the resonant term is nonzero, the twist vanishes in the neighborhood of the tripling bifurcation. We will do this by assuming that $\varepsilon=\omega-m / n \neq 0$ in Eq. (4) and Eq. (7), so that they can 
be transformed to Birkhoff normal form. This gives an expression for the twist that diverges at resonance. The twist, however, is well defined away from resonance, and we will see that $\tau_{0}=0$ at some $\omega=\omega_{0}$ in the neighborhood of $1 / 3$. At this rotation number a twistless torus is created at the origin. As $\omega$ moves away from $\omega_{0}$, the twistless torus grows, corresponding to an extremum in $\Omega(J)$. Again, since $\Omega$ is only defined for the truncated normal form, the existence of the twistless torus is also only guaranteed for this approximation. In the full system the twistless torus will only exist for the Cantor set of values for which its frequency is sufficiently irrational.

As Moser showed [11], the twist for the Hénon map vanishes at $\omega_{0} \approx 0.29$. We show in addition that the twistless circle moves away from the origin as $\omega$ moves towards $1 / 3$. This implies, for example, that when the rotation number of the twistless circle passes through a low order rational number, such as $3 / 10$, a reconnection bifurcation $[8,7]$ should occur. The dynamical consequences of this have already been observed in $[18,16]$, though without explaining their genericity. Using our calculation of the higher order twist, we will obtain a good approximation for the position of this bifurcation.

More generally, the twist can be forced to vanish at any rotation number if there are a sufficient number of parameters. For example, for a cubic map, we will show that with the choice of two parameters we can make $\tau_{0}$ vanish at $\omega=1 / 5$ or $2 / 5$, which results in the instability of the elliptic point at the bifurcation. Similarly the seventh order resonance can be generically destabilized in a three parameter family of quartic maps. The instability of these resonant twistless maps is proved in the appendix.

\section{Resonant Hamiltonian flows}

In this section we will take advantage of the fact that the resonant normal form Eq. (4) is integrable, to obtain exact expressions for the twist near resonance. We begin by rewriting Eq. (4) in the form

$$
\begin{aligned}
H(I, \phi) & =P(I)+Q(I) \cos (n \phi), \quad \text { with } \\
P(I) & =\varepsilon I+A I^{2}+\ldots \\
Q(I) & =I^{n / 2}(B+\ldots), \quad n>2,
\end{aligned}
$$

where the dots denote finite polynomials in $I$. Our goal is to transform $I$ to the true action variable, $J \equiv \frac{1}{2 \pi} \oint I \mathrm{~d} \phi$ for Eq. (8) and obtain the expression 
for the rotation number $\Omega(J)$. To do this, we assume explicitly that $\varepsilon \neq 0$ so that $P$ has a first order zero at the origin.

To find the period, $T(h)$, we solve $H(I, \phi)=h$ for $\phi$ and substitute this into the differential equation for $I$ to obtain

$$
T(h)=\oint \frac{\mathrm{d} I}{\sqrt{Q^{2}-(h-P)^{2}}} .
$$

Here we have taken into account that the extrema in $I$ are visited $n$ times for a full turn of $\phi$; therefore the integral has been multiplied by $n$. D The action is given by the area under the curve $I(h, \phi)$, but it is better to obtain it by integrating $-\phi(I, h) \mathrm{d} I$, because otherwise we would need to solve a quartic or higher equation for $I$. The area under $I$ is given by the difference between the maximum area $2 \pi I_{\max }$ and $2 n$ times the area under the curve $\phi$ between extrema, since the cosine is even, and each extremal is visited $n$ times in one loop. Hence the action turns out to be

$$
J(h)=\frac{1}{2 \pi}\left(2 \pi I_{\max }(h)-2 n \int_{I_{\min }(h)}^{I_{\max }(h)} \phi(I, h) \mathrm{d} I\right) .
$$

As usual one can verify that $T(h)=2 \pi J^{\prime}(h)$, since the boundary terms cancel.

As expected, the period is an Abelian integral of the first kind because $P(I)$ and $Q(I)^{2}$ are polynomials in $I$. The action can also be turned into an Abelian integral by partial integration of Eq. (9). Again the boundary term cancels and we obtain

Lemma 1. The action $J(h)$ and the period $T(h)$ of the resonant normal form Hamiltonian Eq. (8) are Abelian integrals on the Riemann surface

$$
\Gamma: y^{2}=R(z)=Q(z)^{2}-(h-P(z))^{2} .
$$

They are given by

$$
\begin{aligned}
2 \pi J(h) & =\oint_{\gamma} \frac{P^{\prime}(z) Q(z)+Q^{\prime}(z)(h-P(z))}{Q(z) y} z \mathrm{~d} z \\
T(h) & =\oint_{\gamma} \frac{1}{y} \mathrm{~d} z,
\end{aligned}
$$

where $\gamma$ corresponds to the real cycles of $\Gamma$. 
The degree of $R(z)$ is at least $n$ because the lowest order term in $Q(z)$ is $z^{n / 2}$. For $n=3$ the integrals are elliptic, for larger $n$ they are hyperelliptic. The equilibrium points of Eq. (8) correspond to the double roots of $R(z)$. The values of $h$ for which this occurs are called critical values and they can be determined by the discriminant of $R(z)$. The simplest critical value is $h=0$, since there is always a double root at $z=0$ :

$$
\left.R(z)\right|_{h=0}=Q(z)^{2}-P(z)^{2}=-z^{2} \varepsilon^{2}(1+\ldots) .
$$

This corresponds to the fixed point at the origin. Since $R^{\prime \prime}(0)<0$ at $h=0$ there is a maximum between the colliding roots so this corresponds to a vanishing real cycle $\gamma \cdot{ }^{1}$ All that is left from the Abelian integral in this case is the residue of the pole at $z=0$. The limiting period is therefore given by

$$
T(0)=2 \pi i \operatorname{Res}_{z=0} \frac{1}{y}=\frac{2 \pi}{\varepsilon}
$$

This is a trivial result, because $\varepsilon$ was designed to be the deviation in rotation number from $m / n$ in the first place. Note, however, that the original variable $I$ was not the true action. It is the fact that the resonant term is of order $n / 2$ that the frequency at $h=0$ stays the same. A similar calculation will lead to the twist of the origin, which can be changed by the resonant term if $n=3$.

\subsection{Tripling bifurcation for flows}

For the tripling bifurcation the Hamiltonian, from Eq. (4), through quadratic order is

$$
H(I, \phi)=\varepsilon I+A I^{2}+B I^{3 / 2} \cos (3 \phi) .
$$

In general $A$ and $B$ will be functions of $\varepsilon$. With the (generic) assumption that the constant term in the Taylor series of $A(\varepsilon)$ and $B(\varepsilon)$ does not vanish and that $B(\varepsilon) \neq 0$ we can eliminate both parameters in $H$ upon defining

$$
\tilde{I}=\frac{A^{2}}{B^{2}} I, \quad \tilde{h}=\frac{A^{3}}{B^{4}} h, \quad \tilde{\varepsilon}=\frac{A}{B^{2}} \varepsilon, \quad \text { and } \tilde{\phi}=\phi+\psi,
$$

\footnotetext{
${ }^{1} R^{\prime \prime \prime}(0) \neq 0$ : for $n=3$ it vanishes at $\varepsilon=B^{2} / 2 A$, but then $R^{(4)}(0)=-24 A^{2}<0$.
} 
where $\psi=0$ if $A B>0$ and $\psi=\pi / 3$ otherwise. This scaling leaves only the two essential parameters $(\tilde{\varepsilon}, \tilde{h})$, so that the energy equation becomes

$$
\tilde{h}=\tilde{\varepsilon} \tilde{I}+\tilde{I}^{2}+\tilde{I}^{3 / 2} \cos (3 \tilde{\phi}) .
$$

The corresponding elliptic curve is

$$
R(z)=z^{3}-\left(\tilde{h}-\tilde{\varepsilon} z-z^{2}\right)^{2} .
$$

We consider $\tilde{\varepsilon}$ as the bifurcation parameter and the energy $\tilde{h}$ is the parameter selecting a particular torus.

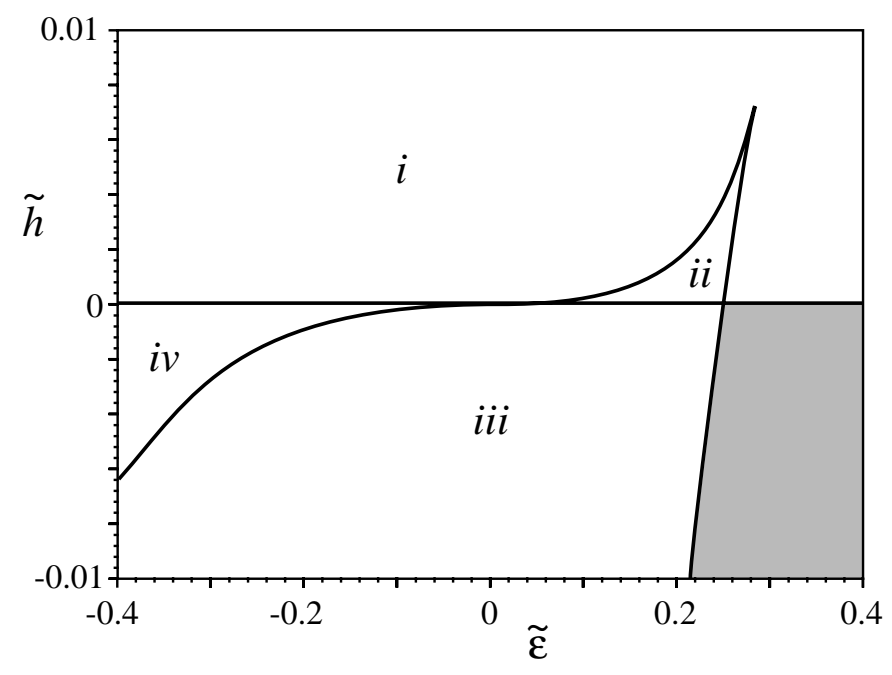

Figure 1: Bifurcation diagram for Eq. (12). The horizontal axis corresponds to the fixed point at the origin. The upper of the two remaining curves represents the saddle 3 -fold orbit and the lower one the elliptic 3 -fold orbit. At $\tilde{\varepsilon}=9 / 32$, where the two lines meet, these orbits collide in a saddle-center bifurcation. The shaded area is energetically forbidden for motion in real phase space.

The phase portrait in the cartesian coordinates Eq. (5), always has one equilibrium point at the origin corresponding to the line $\tilde{h}=0$ in Fig. 1 . The other equilibria are easily found from the zeros of the discriminant $D$ of $R(z)$, which is given by

$$
D(\tilde{h}, \tilde{\varepsilon})=-\tilde{h}^{3}\left[256 \tilde{h}^{2}+\left(27-144 \tilde{\varepsilon}+128 \tilde{\varepsilon}^{2}\right) \tilde{h}+4 \tilde{\varepsilon}^{3}(-1+4 \tilde{\varepsilon})\right] .
$$


Apart from the triple zero at $\tilde{h}=0, D$ has two distinct real roots for $\tilde{h}$ when $\tilde{\varepsilon}<9 / 32$. These are the energy levels for one of the 3 -fold equilibria which correspond to the period three orbits in the original, nonrotating frame. The point $(\tilde{\varepsilon}, \tilde{h})=\left(9 / 32,(3 / 16)^{3}\right)$ is the cusp in Fig. 1 , where the period three orbits collide. There are four regions in the bifurcation diagram; the corresponding phase portraits are shown in Fig. 2. In region (i) there is one interval of positive $R$ corresponding to one interval of real momenta, hence one torus. Crossing the critical lines to regions (ii) and (iv) creates a second interval of positive $R$ out of a double root. Entering region (iii) from these regions by crossing another line of critical points destroys a positive interval in a double root so again there is only one torus corresponding to each point in region (iii). Regions (i) and (iii) can also be left by destroying the one positive interval in a double root so that no motion at all is possible (the shaded region).

The details of which torus in the phase portrait belongs to what region are shown in Fig. 2. Consider the one positive interval of region (i): For $\tilde{\varepsilon}<0$ it corresponds to tori sufficiently far away from the origin (part of dotted line); for $0<\tilde{\varepsilon}<1 / 4$ it corresponds to all tori outside the separatrix (dotted line); for $1 / 4<\tilde{\varepsilon}<9 / 32$ it corresponds to all tori outside the separatrix (dotted line) and also to tori sufficiently close to the origin (part of solid line); for $\tilde{\varepsilon}>9 / 32$ it corresponds to all tori (dotted line). For the other regions the correspondance is analogous. The boundaries of the regions, where two roots of $R$ coalesce, correspond to the equilibrium solutions. Note that the origin is a minimum of $H\left(\left(q^{2}+p^{2}\right) / 2\right.$, arctan $\left.p / q\right)$ for positive $\tilde{\varepsilon}$ and a maximum for negative $\tilde{\varepsilon}$, showing that it is stable when $\tilde{\varepsilon} \neq 0$. Also note that there is no change in the dynamics when $\tilde{\varepsilon}$ crosses the value $1 / 4$.

\subsection{The twist}

The twist $\tau(h)$ of a torus with energy $h$ is given by Eq. (3), thus

$$
\tau(h)=\frac{\partial \Omega}{\partial J}=\frac{\Omega^{\prime}(h)}{J^{\prime}(h)}=-\frac{J^{\prime \prime}(h)}{J^{\prime}(h)^{3}} .
$$

or, expanding near the origin in a series in $J$, we have

$$
\tau(J)=-\varepsilon^{3} J^{\prime \prime}(0)+\varepsilon^{4}\left[3 \varepsilon J^{\prime \prime}(0)^{2}-J^{\prime \prime \prime}(0)\right] J+O\left(J^{2}\right) .
$$

To compute these first two terms in the twist, we must find the second and third derivatives of $J(h)$ at $h=0$. 


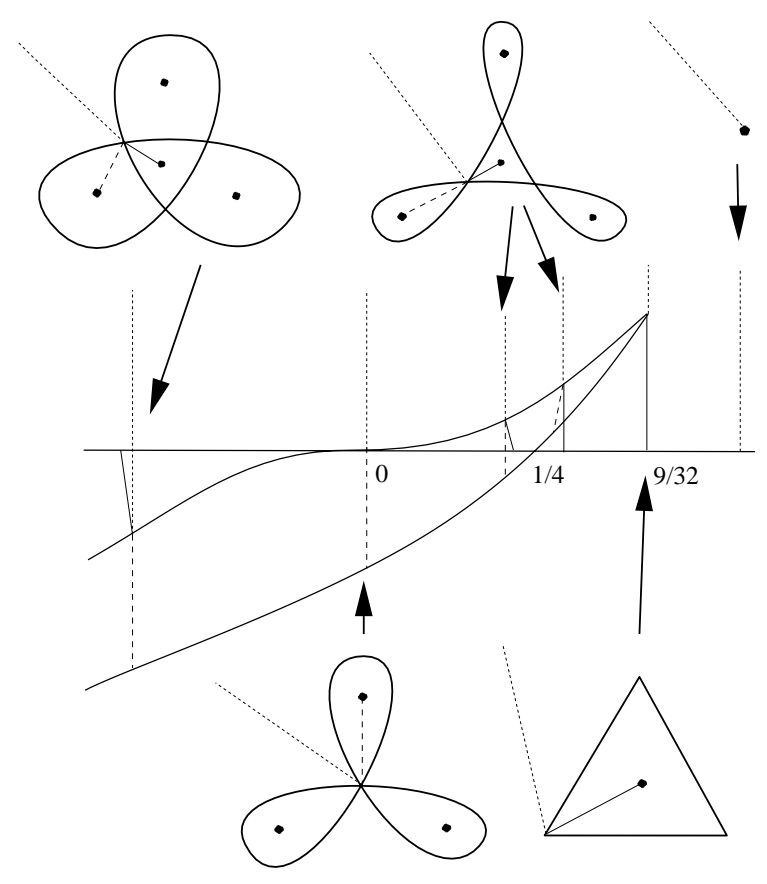

Figure 2: Sketch of the bifurcation diagram for the tripling bifurcation in $(\tilde{\varepsilon}, \tilde{h})$. The thick curves represent the energy levels of the equilibrium points. The phase portraits correspond to fixed values of $\tilde{\varepsilon}$, and the thin, vertical lines represent the energy ranges for the topologically distinct families of tori, shown in each phase portrait. Each group of thin lines corresponds to a single $\tilde{\varepsilon}$, but they are drawn slightly skewed for clarity.

Lemma 2. The twist of the fixed point at the origin of the Hamiltonian Eq. (11) is given by Eq. (3) where

$$
\begin{aligned}
\tau_{0} & =2 A-\frac{3 B^{2}}{2 \varepsilon} \\
\tau_{1} & =\frac{3}{2} \frac{B^{2}}{\varepsilon^{3}}\left(8 \varepsilon A-3 B^{2}\right) .
\end{aligned}
$$

Proof. Upon manipulation of Eq. (10), we can reduce the integrals to calcu- 
late to

$$
\begin{aligned}
J^{\prime \prime}(h) & =\frac{1}{2 \pi} \oint \frac{h-P(z)}{y^{3}} d z \\
J^{\prime \prime \prime}(h) & =\frac{1}{2 \pi} \oint\left(\frac{3 Q^{2}}{y^{5}}-\frac{2}{y^{3}}\right) d z .
\end{aligned}
$$

To evaluate the twist we need to compute these integrals at $h=0$. Since the cycles reduce to loops around the origin, this amounts to computing residues of the integrands.

Changing the Hamiltonian by adding higher order terms in $P$ or $Q$, or adding more trigonometric terms with amplitudes at least $I^{5 / 2}$ will change the coefficient of $z^{4}$ and higher order terms. However, this coefficient does not influence the residue of $J^{\prime \prime}$, so that the twist $\tau_{0}$ is not changed by these terms. The next order twist $\tau_{1}$ can, however, be changed. The complete second twist is calculated for maps in the next section.

A similar calculation for $n>3$ in Eq. (4), shows that the lowest order twist $\tau_{0}$ is independent of $B$. Thus it vanishes only if the bare twist term, $A=0$. This shows that the tripling normal form is the only one for which the twistless torus is generically created at the origin. The reason that the tripling is different is that the order of $Q(I)$ is smaller than that of the twist term $A I^{2}$.

Lemma 2 implies that the twist vanishes at the origin when

$$
\varepsilon=\varepsilon_{0} \equiv \frac{3 B^{2}}{4 A},
$$

which corresponds to the frequency, in the nonrotating frame, of

$$
\omega_{0}=\frac{1}{3}+\frac{3 B^{2}}{4 A} \text {. }
$$

The twistless curve moves away from the origin as $\varepsilon$ moves towards zero, corresponding to approaching the tripling bifurcation. Using these expressions, we can compute the rotation number of the twistless curve near $\varepsilon_{0}$ where it is created. In the original nonrotating frame, the rotation number is given by

$$
\Omega_{0}(\omega)=\omega-\frac{1}{3} \frac{A}{B^{2}}\left(\omega-\omega_{0}\right)^{2}+O\left(\omega-\omega_{0}\right)^{3},
$$

where we have expressed it as a function of the rotation number $\omega$ of the origin. The twistless torus can be seen in Fig. 3, which is a contour plot 
of the rotation number on the bifurcation diagram. Since $\Omega^{\prime}(h)=0$ at the twistless torus, it corresponds to the locus of points where the contours are vertical. The twistless torus emerges from the origin at $\tilde{\varepsilon}=3 / 4$, and collides with the saddle-center bifurcation at $\tilde{\varepsilon}=9 / 32$. In region (ii) the plotted curves correspond to the rotation number of the torus in the central triangle; in region (iv) they give the rotation number of the torus in the outer region. In this way the rotation number can be made continuous. The torus in the central triangle corresponding to region (iv) has the negative rotation number of the one in the outside region with the same $h$. This is a result of the sum rule $\oint_{\gamma_{1}+\gamma_{2}} \frac{\mathrm{d} z}{y}=0$, where the $\gamma_{i}$ are the two real cycles for $n=3$. In region (iii) the rotation number (in the rotating frame!) of the tori surrounding the 3 stable fixed points is shown. In the non-rotating frame the whole island would have rotation number $1 / 3$ with respect to the central fixed point. In the rotating frame, however, this rotation number measures the winding around each of the respective three fixed points.

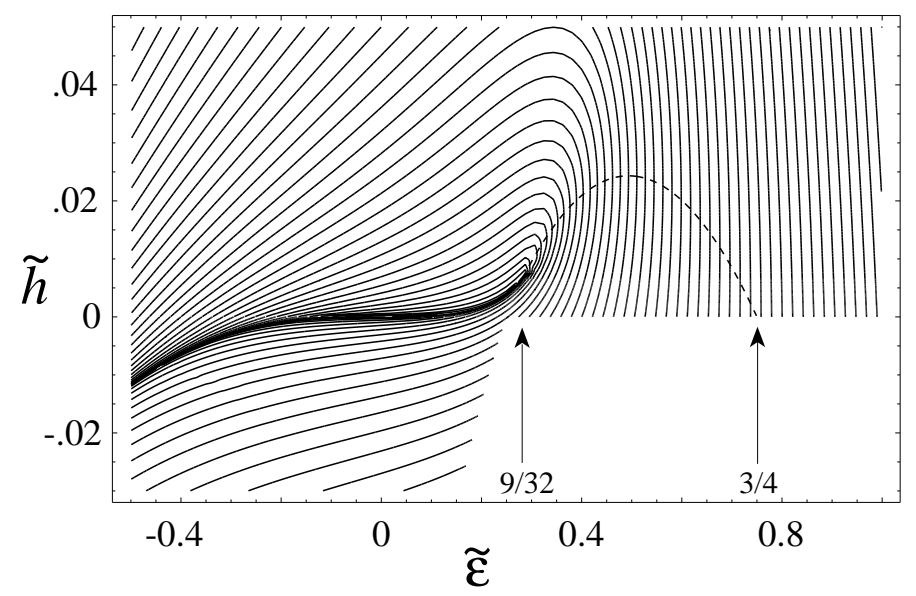

Figure 3: Lines of constant rotation number spaced equidistant with $\Delta \Omega=$ 0.02 on the bifurcation diagram for the tripling bifurcation. The contours cluster around the line of the unstable 3 -fold orbit, because there the period $T$ diverges logarithmically.

Since the expression for $\tau_{0}$ is not affected by higher order terms in $P$ or $Q$, we can describe the generic scenario of the tripling bifurcation as follows. A sufficient distance from the tripling bifurcation, when $\tilde{\varepsilon}>3 / 4$, the periodic orbit has twist, i.e., the rotation number is a monotone function of the transverse action near the orbit. Note that this corresponds to $\omega>1 / 3$ if the 
rotation number increases with action, i.e., $A>0$, otherwise it corresponds to $\omega<1 / 3$. As $\omega$ moves towards $1 / 3$ or equivalently as $\tilde{\varepsilon}$ decreases toward zero, but before there is any obvious sign of the $1 / 3$ bifurcation, the central periodic orbit loses its twist at the parameter value $\tilde{\varepsilon}=3 / 4$.

Beyond that point the twist in the island is no longer monotone, and there is a twistless torus somewhere in the island. For $\tilde{\varepsilon}<3 / 4$ a generic perturbation of $H$ cannot destroy a diophantine twistless curve with nonzero second twist, as is shown in [5]. The difference with ordinary KAM theory is that for small perturbations the twistless torus with the same rotation number exists not necessarily for the same but for slightly changed parameter $\tilde{\varepsilon}$. As $\tilde{\varepsilon} \rightarrow 9 / 32^{+}$the twistless torus reaches rotation number $\omega=1 / 3$ and there is a saddle-center bifurcation creating a pair of period three orbits at nonzero radius in the island. The twist inside the central triangle is opposite to that outside the period three island chain. Beyond this point the twistless torus is replaced by the separatrix with rotation number $1 / 3$. Of course, the separatrix is generically a homoclinic tangle.

Eventually, when $\omega=1 / 3$ or $\tilde{\varepsilon}=0$, the saddle period three orbit collides with the central periodic orbit. Moving past this point, the saddle period three orbit re-emerges, and the origin has changed the sign of its twist. Thus for $\tilde{\varepsilon}<0$, the rotation number is again a monotone increasing function.

We conclude by stating this more formally

Theorem 3. Near a tripling bifurcation of a periodic orbit, a two degree of freedom Hamiltonian system has a codimension one twistless bifurcation, corresponding to the vanishing of the twist at the periodic orbit. For the normal form, Eq. (11), this results in the creation of a twistless curve at $\tilde{\varepsilon}_{0}=3 / 4, E q$. (14), that moves away from the origin as the tripling point is approached, and eventually collides with the saddle-center bifurcation of the period three orbits.

Note that the coefficients of the original normal form, Eq. (8) can be taken to be constants only when $\varepsilon$ is sufficiently small, or equivalently, $\omega$ is close to $1 / 3$. However, we can always transform a Hamiltonian with an elliptic fixed point into Eq. (8) as long as $\omega \neq 0,1 / 2$. Also higher order terms can be removed assuming that the specific $\omega$ is nonresonant to that order. In this case, we need not assume that $\varepsilon$ is small; however, the coefficients of the normal form will depend upon $\varepsilon$. In this case Eq. (11) is valid sufficiently close to the origin, and our scaling to obtain Eq. (12) is valid provided only that the coefficients $A$ and $B$ do not vanish over the desired range of $\varepsilon$. 
The statement about the creation of the twistless curve is only based on a calculation near the origin, and therefore valid. It might of course happen that a given one parameter family of Hamiltonians never reaches the creation of the twistless curve, because e.g., the frequency of the fixed point is not a monotonous function of the parameter (it might, e.g., not even reach the $1 / 3$ resonance). The global fate of the twistless curve as stated in the second sentence of the theorem might be changed by higher order terms that are not removable, e.g. close to the $2 / 5$ or $2 / 7$ resonance. This is why we only claim that this behavior occurs in the normal form and not necessarily in the original Hamiltonian.

\section{$3 \quad$ Tripling bifurcation for area-preserving maps}

In this section we study the normal form for an area-preserving map, $f$, in the neighborhood of an elliptic fixed point with a tripling bifurcation. We will show that the twist generically vanishes in the neighborhood of this bifurcation.

Suppose that $f$ has an elliptic fixed point at the origin with rotation number $\omega$, i.e., with linear multipliers $\lambda$ and $\bar{\lambda}$, Eq. (6). We can put the linearization of the map in the complex, diagonal normal form $(z, \bar{z}) \mapsto(\lambda z, \bar{\lambda} \bar{z})$ by introducing a pair of complex variables $(z, \bar{z})$ defined by the linear transformation

$$
(x, y)^{T}=\frac{1}{2 i}(v z-\bar{v} \bar{z}),
$$

where $v$ is the complex eigenvector of $D f(0)$ associated with $\lambda$. If we choose the normalization of the eigenvectors so that $\bar{v} \times v=2 i$, then the $z$ variables are related to action-angle variables by Eq. (5). In the new variables, the map has the power series

$$
z \mapsto \lambda\left[z+\sum_{n=2}^{r} \sum_{j=0}^{n} a_{j, n-j} z^{j} \bar{z}^{n-j}\right]+O(r+1),
$$

where the terms are ordered as homogeneous monomials of degree $n$ in $z$ and $\bar{z}$. The map for $\bar{z}$ is simply the complex conjugate of Eq. (16). Note that the complex coefficients, $a_{j k}$, are not all independent when the map is area-preserving. 
The normal form for Eq. (16) can be computed by applying a sequence of canonical transformations to eliminate as many terms in the series as possible [13]. As is well known each of the coefficients $a_{j k}$ can be formally eliminated providing the multiplier does not satisfy a "resonance" condition of the form

$$
\lambda^{j-k-1}=1 .
$$

In particular for any $\lambda$, the terms with $j-k=1$ cannot be eliminatedthese we call the "twist" terms, since they give rise to shear in the rotation about the fixed point. These terms give rise to the Birkhoff normal form Eq. (2). However, if $\lambda$ is a root of unity, then other resonant terms occur. For example, when $\omega=1 / 3$, hence by (6) $\lambda=\exp (2 \pi i / 3)$, then we cannot eliminate terms for which $j-k=1 \bmod 3$. In this case the resonant normal form is

$$
z \mapsto \lambda\left[z+a_{02} \bar{z}^{2}+a_{21} z^{2} \bar{z}+a_{13} z \bar{z}^{3}+a_{40} z^{4}+a_{32} z^{3} \bar{z}^{2}+a_{05} \bar{z}^{5}\right]+O(6)
$$

To study the dynamics in the neighborhood of the tripling bifurcation, we assume that the lowest order resonant term, $a_{02}$, is nonzero. In this case we can scale $z$ to eliminate this coefficient. When the map is area-preserving, the thirteen real coefficients in the resonant normal form must satisfy four conditions through fifth order. After applying these conditions and scaling the map to eliminate the coefficient $a_{02}$, we can reduce the resonant normal form to a seven parameter family through 5 th order

$$
\begin{aligned}
z \mapsto \lambda[z & +\bar{z}^{2}+(1+i \alpha) z^{2} \bar{z}+(\beta+i \gamma) z \bar{z}^{3} \\
& \left.+a_{40} z^{4}+(\delta+i \eta) z^{3} \bar{z}^{2}+a_{05} \bar{z}^{5}\right]+O(6) .
\end{aligned}
$$

The real coefficients $\alpha, \beta, \gamma, \eta$ and the complex coefficient $a_{05}$ are arbitrary and

$$
a_{40}=\frac{1}{4}[2+\beta+i(2 \alpha-\gamma)], \quad \delta=2 \beta-\frac{1+\alpha^{2}}{2} .
$$

The third power of this map can be approximated (to any desired finite order) by the time $2 \pi$ map of a Hamiltonian, which gives the connection to the previous section.

Taking the resonant form Eq. (18) as our model, we imagine that the coefficients are functions of $\omega$-constants in the simplest case - and that $\omega$ is the bifurcation parameter. When $\omega \neq 1 / 3$ the resonant form can be transformed to Birkhoff normal form. In fact if $\omega$ is not $1 / 4,1 / 5$ or $2 / 5$, we 
can reduce the map to Birkhoff normal form through fifth order. Our goal is to compute the twists $\tau_{0}$ and $\tau_{1}$ for this map. As already noted in the end of the last section the transformation to this particular form is possible even far away from the resonant case. Even though the 1/3-resonant term could be removed when $\omega \neq 1 / 3$, it does not have to be removed. At least close to the origin and away from other low order resonances the above normal form therefore is a good approximation for a large neighborhood of $\omega=1 / 3$.

To carry out the transformation we must apply a sequence of four, near identity coordinate transformations to eliminate successively all of the quadratic, cubic, quartic and quintic terms except for the twist terms. One way to obtain area-preserving coordinate transformations is to use a canonical generating function of the form

$$
P q+F^{(r)}(P, q)
$$

where $F^{(r)}$ is a homogeneous polynomial of degree $r+1$. This implicitly generates a canonical transformation through the equations

$$
p=P+\frac{\partial F^{(r)}}{\partial q}(P, q) \quad, \quad Q=q+\frac{\partial F^{(r)}}{\partial P}(P, q) .
$$

The lowest order terms of the transformation $(q, p) \mapsto(Q, P)$ are easily obtained explicitly

$$
P=p-\frac{\partial F^{(r)}}{\partial q}(p, q)+O(2 r-1), \quad Q=q+\frac{\partial F^{(r)}}{\partial p}(p, q)+O(2 r-1)
$$

and the higher order terms can be obtained with more effort, order-by-order. Converting the transformation to complex coordinates, with $Z=P+i Q$, yields a transformation of the form

$$
Z=z+\sum_{i=0}^{r} b_{i, r-i} z^{i} \bar{z}^{r-i}+O(2 r-1)
$$

where the coefficients $b_{i j}$ can be explicitly computed in terms of the coefficients of $F^{(r)}$.

We wish to compute the nonresonant Birkhoff normal form for Eq. (18) through fifth order. To do this, we first apply the canonical transformation 
$F^{(2)}$, with the coefficients chosen to eliminate the quadratic term in Eq. (18). This is possible whenever $\omega \neq 1 / 3$. This transformation generates terms of cubic and higher order in the map. Whenever $\omega \neq 1 / 4$ we can then apply a transformation generated by $F^{(3)}$ to eliminate all of the cubic terms, except for the twist term $z^{2} \bar{z}$. This transformation will not modify the quartic terms in the map, but because of the $O(5)$ correction terms in Eq. (19), it will modify the quintic terms. Finally, whenever $\omega \neq 1 / 5$ or $2 / 5$, we can apply transformations $F^{(4)}$ and $F^{(5)}$ to eliminate all of the quartic and all of the quintic terms except for the twist $z^{3} \bar{z}^{2}$. These transformations will not modify the coefficient of the twist term, however, and therefore we do not need to compute them in order to find the Birkhoff normal form to fifth order. This yields the Birkhoff normal form for Eq. (18) in the form of Eq. (2) where

$$
\begin{aligned}
& \pi \tau_{0}=\alpha-\frac{\left(3 t^{2}-1\right)}{t\left(t^{2}-3\right)} \\
& \pi \tau_{1}=4(\eta-\gamma)+12 \frac{3 t^{2}-1}{t\left(t^{2}-3\right)}\left(\frac{\left(t^{2}+1\right)^{3}}{2 t^{2}\left(t^{2}-3\right)^{2}}+\pi \tau_{0} \frac{3 t^{2}-1}{t\left(t^{2}-3\right)}-\beta\right)
\end{aligned}
$$

with $t \equiv \tan (\pi \omega)$.

Near $\varepsilon=0$ we can reduce these expressions to

$$
\begin{aligned}
& \pi \tau_{0}=-\frac{1}{3 \pi \varepsilon}+\alpha+O(\varepsilon) \\
& \pi \tau_{1}=\frac{2}{9 \pi^{3} \varepsilon^{3}}+\frac{4}{3 \pi \varepsilon^{2}} \tau_{0}-\frac{4}{\pi \varepsilon} \beta+4\left(\eta-\gamma-2 \tau_{0}\right)+O\left(\varepsilon^{1}\right) .
\end{aligned}
$$

The dominant terms in these expressions, though they look quite different, are actually equivalent to those obtained for the flow in Lemma 2 providing we set $\beta=\eta=\gamma=0$. $^{2}$ Nonzero values would correspond to higher order terms in the flow Hamiltonian.

The function $\tau_{0}(\omega)$ in Eq. (20) is shown in Fig. 4. It is monotone increasing and maps the domain $\left[\frac{1}{6}, \frac{1}{3}\right) \cup\left(\frac{1}{3}, \frac{1}{2}\right)$ one-to-one onto $(-\infty, \infty)$. Moreover, the coordinate transformations that we carried out to compute $\tau_{0}$ are valid

${ }^{2}$ The time $2 \pi$ map of the flow of Eq. (11), is approximately

$$
\zeta^{\prime} \mapsto \lambda\left(\zeta+2 \pi i A|\zeta|^{2} \zeta+\frac{3 \pi i B}{\sqrt{2}} \bar{\zeta}^{2}\right)
$$

which is equivalent to Eq. (18), upon identifying $\alpha=\frac{2 \pi A}{s}$, where $s=(3 \pi B)^{2} / 2$ is the factor that scales the action to normalize $a_{02}$ in the map. 
in this domain. Thus for any $\alpha$, there is a rotation number $\omega_{0}$ for which $\tau_{0}\left(\omega_{0}\right)=0$. If $\omega_{0}$ is not $1 / 5,1 / 4$ or $2 / 5$ then the transformations leading to the expression for $\tau_{1}$ are valid. Whenever $\tau_{1}\left(\omega_{0}\right) \neq 0$, as is generically true for our expressions, the Moser twist theorem [13] implies that there are invariant circles in the neighborhood of the elliptic point. Thus we can conclude

Theorem 4. Let $f_{\omega}$ be area-preserving map with an elliptic fixed point that has rotation number $\omega$. Suppose that when $f$ is transformed to its resonant normal form, Eq. (18), the coefficient $\alpha$ is finite (i.e. that $a_{02}(\omega) \neq 0$ ). Then there is an $\omega_{0} \in\left[\frac{1}{6}, \frac{1}{3}\right) \cup\left(\frac{1}{3}, \frac{1}{2}\right)$ such that $f_{\omega_{0}}$ has a twistless bifurcation, i.e. where $\tau_{0}\left(\omega_{0}\right)=0$, from Eq. (20). The elliptic fixed point is stable at $\omega_{0}$ whenever $\omega_{0} \notin\{1 / 5,1 / 4,2 / 5\}$, and $\tau_{1}\left(\omega_{0}\right) \neq 0$, as is generically the case.

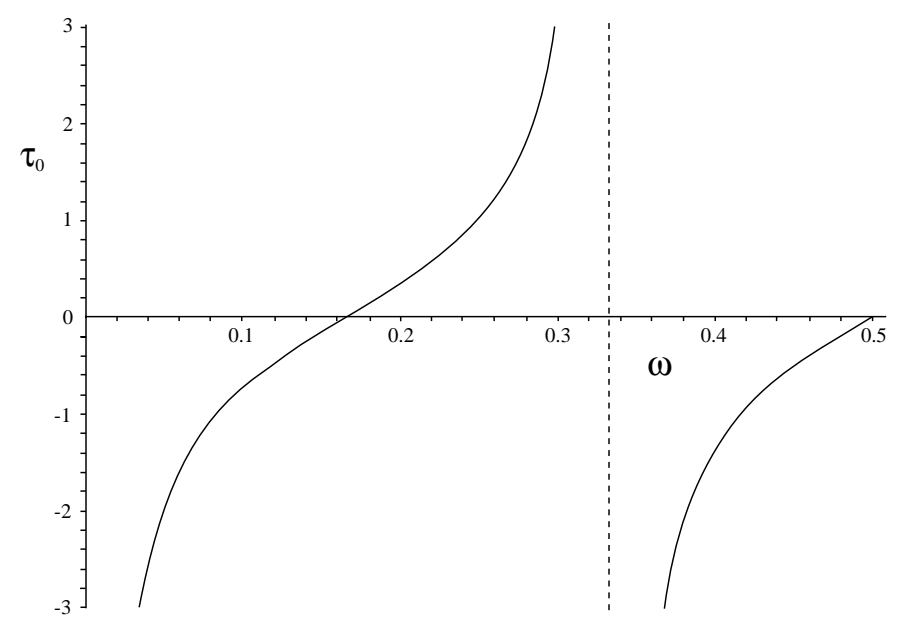

Figure 4: Plot of the twist $\tau_{0}(\omega)$ in Eq. (20) with $\alpha=0$.

Note that in Eq. (21) the dominant contribution to $\tau_{1}\left(\omega_{0}\right)$, when $\omega_{0}$ is reasonably close to $\frac{1}{3}$, has the $\operatorname{sign}$ of $\varepsilon$. Thus there are two typical scenarios for the twistless bifurcation. If $\omega_{0}<\frac{1}{3}$, then typically $\tau_{1}\left(\omega_{0}\right)<0$, and so the rotation number $\Omega(J)$ is locally monotone decreasing as $\omega \rightarrow \omega_{0}$ from below. The twistless curve is created at a local maximum of $\Omega(J)$ which moves away from the origin as $\omega$ approaches $\frac{1}{3}$. Conversely, if $\omega_{0}>\frac{1}{3}$, then typically, $\tau_{1}\left(\omega_{0}\right)>0$, so the rotation number is monotone increasing as $\omega \rightarrow \omega_{0}$ from above. The twistless curve corresponds to a local minimum of $\Omega(J)$ which moves away from the origin as $\omega$ moves towards $\frac{1}{3}$. On the other hand, it is also possible that $\tau_{1}\left(\omega_{0}\right)$ has the opposite sign of $\varepsilon$, in which case 
the bifurcation creates a twistless curve as $\omega$ moves from $\omega_{0}$ in the direction away from $\frac{1}{3}$.

In the actual dynamics we expect that when the rotation number of the twistless curve passes through rational points, there will be reconnection bifurcations $[8,7]$ in which two island chains with the same rotation number annihilate each when they collide at the twistless "curve." We expect this to happen often for the island chains with rotation numbers in the interval $\left[\omega_{0}, \frac{1}{3}\right)$, because after birth, they will travel outward from the fixed point, and if they move rapidly enough will cross the twistless curve.

In the next sections, we will give examples of the twistless bifurcation for the Hénon map, and for a quartic map, where we can choose parameters so that $\omega_{0}$ is a low order rational.

\subsection{Tripling bifurcation for the Hénon map}

Any quadratic, area-preserving map of the plane can be written in the form $[6]$

$$
(x, y) \mapsto\left(y-k+x^{2},-x\right)
$$

It has an elliptic fixed point at $x_{e}=-y_{e}=1-\sqrt{1+k}$ when $-1<k<3$. The rotation number at the fixed point is

$$
\omega=\frac{1}{\pi} \arcsin \left(\frac{1+k}{4}\right)^{1 / 4} .
$$

The normalized, complex eigenvector is $v=\frac{1}{\sqrt{s}}(-\lambda, 1)^{T}$, where $s=\sin (2 \pi \omega)$. Using Eq. (15), shifted to the elliptic fixed point, we define the complex variable

$$
z=-\frac{1}{\sqrt{s}}\left(x-x_{e}+\bar{\lambda}\left(y-y_{e}\right)\right),
$$

so that the Hénon map becomes

$$
z \mapsto \lambda z+\frac{1}{4 s^{3 / 2}}(-\lambda z+\bar{\lambda} \bar{z})^{2}
$$


Applying the normal form transformation reduces the map to the Birkhoff normal form, Eq. (2), with

$$
\begin{aligned}
\tau_{0} & =\frac{1}{2^{6} \pi} \frac{\left(3 t^{2}-5\right)\left(1+t^{2}\right)^{3}}{t^{4}\left(3-t^{2}\right)} \\
\tau_{1} & =\frac{1}{2^{13} \pi} \frac{\left(1+t^{2}\right)^{6}\left(51 t^{10}-637 t^{8}+2038 t^{6}-2706 t^{4}+2055 t^{2}-705\right)}{t^{9}\left(t^{2}-1\right)\left(t^{2}-3\right)^{3}} .
\end{aligned}
$$

where $t=\tan (\pi \omega)$, as before. Moser previously obtained $\tau_{0}$ in $[11]^{3}$. Note that $\tau_{0}$ vanishes only when $t=\sqrt{5 / 3}$, which occurs when $\omega=\omega_{0}=$ $\left.\frac{1}{2 \pi} \arccos \left(-\frac{1}{4}\right)\right) \approx 0.2902153116$, or $k=9 / 16$. As Moser noted, $\omega_{0}$ is a transcendental number, and therefore satisfies a Diophantine condition. According to a theorem of Rüssmann, this implies that there are invariant curves in the neighborhood of the fixed point so it is stable. This result is obtained more easily by noting that $\tau_{1}\left(\omega_{0}\right) \approx-.3366$ is nonzero. In this case the stability of the fixed point follows from the Moser twist theorem [13].

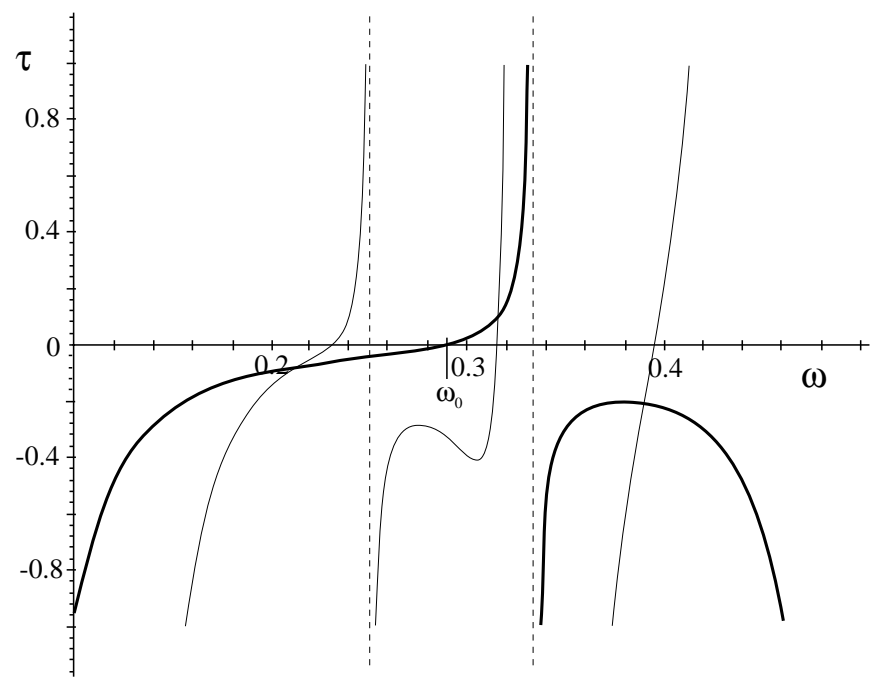

Figure 5: Plot of $\tau_{0}$ (thick line) and $\tau_{1}$ (thin line) for the Hénon map as a function of $\omega$. Here $\tau_{0}$ vanishes only at $\omega \approx 0.2902153116$, and $\tau_{1}$ at $\omega \approx 0.2308206101$, 0.3137515644 , and 0.3944381765 .

Both twist functions are shown in Fig. 5 as a function of $\omega$. The Birkhoff normal form has a curve of zero twist that is born at $\omega_{0}$ and moves outward

\footnotetext{
${ }^{3}$ Moser's equivalent expression is apparently not written in standard canonical variables, and differs from ours by a factor of $-1 / 2$.
} 
in action as $\omega$ increases at least up to 0.31375 where $\tau_{1}$ vanishes. This upper endpoint is artificial, however, since the higher order twists will become increasingly important as $\tau_{1}$ nears zero. The rotation number of the twistless curve is approximately

$$
\Omega_{0}(\omega) \approx \omega-\frac{1}{2} \frac{\tau_{0}^{2}}{\tau_{1}}
$$

This function is shown in Fig. 6. Based on the flow results, we expect that the twistless curve in some sense collides with the saddle-center point of the period three orbit at $k=1$. This corresponds to $\omega=\omega_{5}$ in the figure so that $\Omega_{0}\left(\omega_{5}\right)=1 / 3$. We saw in $\S 2$ that the twistless curve does collide with the saddle-center bifurcation for the resonant normal form of the flow, which was integrable and for which we obtained an exact expression for $\Omega$. The predictions of the normal form for the map, however, are not of much use here because the Hénon map exhibits considerable chaos for orbits at these radii. If the twistless "curve" continues to the tripling, it will no longer be an invariant circle; presumably it will be a cantorus.

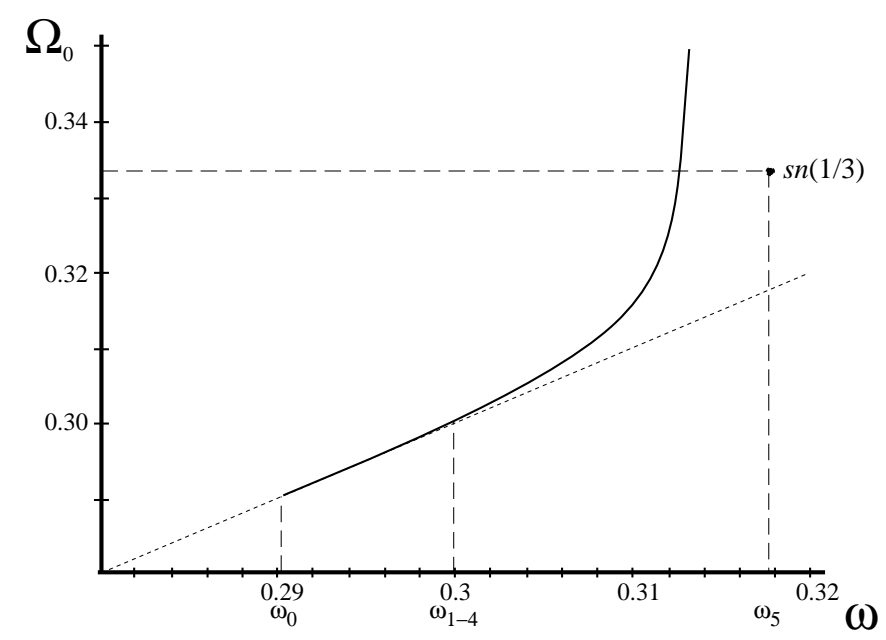

Figure 6: The rotation number of the twistless circle, $\Omega_{0}(\omega)$ near the tripling bifurcation of the Hénon map.

In the interval where the twistless curve exists, $\omega_{0}<\omega<\omega_{5}$, there should be many unusual bifurcations. For example, whenever the twistless curve crosses a rational rotation number, there will typically be a collision 
of two island chains of periodic orbits with this rotation number. The most prominent case corresponds to the lowest order rational in this interval, 3/10, which we sketch in the phase portraits in Fig. 7. Similar bifurcations can be observed when the twistless curve passes through other low order rationals (e.g. 4/13 or 5/16). The normal form indicates that the twistless curve has rotation number $\Omega_{0}(\omega)=3 / 10$, when $\omega \approx 0.2995198$, using the expansion Eq. (22). Translating back to the parameter of the Hénon map, this corresponds to $k \approx 0.7060175$. Indeed there is a saddle-center $3 / 10$ bifurcation near $k=0.7063832$, which is very close to our prediction. However, the situation is more complicated than the normal form would indicate. We list the bifurcations in Table 1, and sketch the corresponding situation in Fig. 8. The four 3/10 orbits are created in two successive 3/10 saddle-center bifurcations at $\omega_{1}$ and $\omega_{2}$. In the interval $\omega_{2}<\omega<\omega_{4}$ there are four 3/10 orbits, two are elliptic and two are hyperbolic. The stable and unstable manifolds of the two hyperbolic 3/10 orbits undergo a reconnection bifurcation [7] near $\omega_{3}$, see Fig. 7. Subsequently the "inner" pair of 3/10 orbits collides with the elliptic fixed point, in a decupling bifurcation at $\omega_{4}=3 / 10$. The outer pair of $3 / 10$ orbits move away from the origin and persist as $k \rightarrow \infty$. These last two orbits are the orbits that we continue from the "anti-integrable" limit, [17].

The word "reconnection" should be clarified. In the integrable approximation $[8,15]$ an actual reconnection occurs at a point, $\omega_{3}$. The Hénon map is of course not integrable, and therefore $\omega_{3}$ is strictly speaking not a point, but rather should be replaced by the interval for which the map has heteroclinic orbits between the two unstable periodic orbits. This interval is extremely small.

\subsection{Normal form for a quartic map}

To study codimension two bifurcations, we need a map with two essential parameters. Since the quadratic map has only one (the rotation number), we turn to a higher order polynomial map which we take to be the composition of a rotation and a shear

$$
\left(\begin{array}{l}
x \\
y
\end{array}\right) \mapsto R_{2 \pi \omega}\left(\begin{array}{l}
x \\
y+x^{2}+c x^{3}+d x^{4}
\end{array}\right)
$$

where $R_{\varphi}$ is a counterclockwise rotation by the angle $\varphi$. This map has 3 essential parameters, $\omega, c$, and $d$. According to our general theory, we expect 

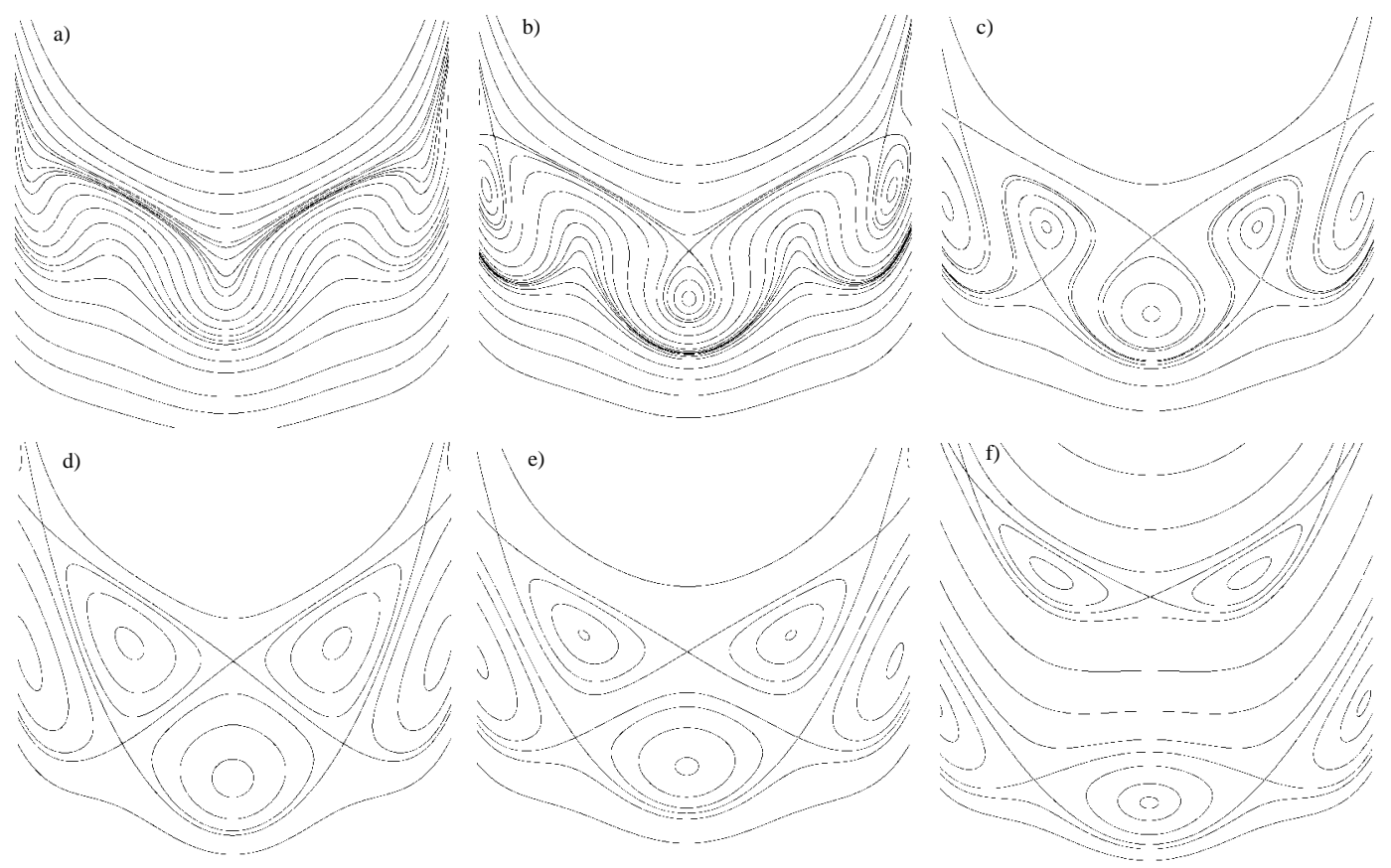

Figure 7: Phase portraits of the Hénon map near $\omega_{3}$ where the $3 / 10$ reconnection bifurcation occurs. From left to right and top to bottom the parameters are $k=0.70638,0.70639,0.70640,0.70640121,0.70642,0.70650$. These parameter values generate representative frequencies in the range $\omega_{0}<\omega<\omega_{4}$ as shown in Table 1.

that with a choice of $c$ we can specify $\omega_{0}$ so that $\tau_{0}=0$ when $\omega=\omega_{0}$. With the proper choice of $d$ we can set $\tau_{1}=0$, as well.

Transforming this map into Birkhoff normal form, we obtain Eq. (2), providing the nonresonance conditions are satisfied as usual. The twists are

$$
\begin{aligned}
\pi \tau_{0}= & \frac{3 c}{8}+\frac{5-3 t^{2}}{8 t\left(t^{2}-3\right)} \\
\pi \tau_{1}= & \frac{-705+2055 t^{2}-2706 t^{4}+2038 t^{6}-637 t^{8}+51 t^{10}}{512 t^{3}\left(t^{2}-3\right)^{3}\left(t^{2}-1\right)} \\
& +\frac{3 c}{256 t\left(t^{2}-1\right)}\left[\frac{-225+556 t^{2}-534 t^{4}+204 t^{6}-17 t^{8}}{t\left(t^{2}-3\right)^{2}}+\frac{c}{2}\left(17-38 t^{2}+17 t^{4}\right)\right] \\
& +\frac{3 d}{16} \frac{7-5 t^{2}}{t\left(t^{2}-3\right)}
\end{aligned}
$$


Table 1: Bifurcation Points for the Hénon map

\begin{tabular}{c|l|l|l} 
Bifurcation & label & $\omega$ & $k$ \\
\hline twistless & $\omega_{0}$ & 0.2902153 & $\frac{9}{16}$ \\
\hline $\operatorname{sn}(3 / 10)$ & $\omega_{1}$ & 0.2995432 & 0.7063832 \\
\hline $\operatorname{sn}(3 / 10)$ & $\omega_{2}$ & 0.2995438 & 0.7063926 \\
\hline reconnection & $\omega_{3}$ & 0.2995444 & 0.70640121 \\
\hline decupling & $\omega_{4}$ & $\frac{3}{10}$ & 0.7135255 \\
\hline $\operatorname{sn}(1 / 3)$ & $\omega_{5}$ & 0.3179717 & 1 \\
\hline $\operatorname{tripling}$ & $\omega_{6}$ & $\frac{1}{3}$ & $\frac{5}{4}$
\end{tabular}

For the cubic map $(d=0)$ we can make $\tau_{0}=0$ at any $\omega_{0} \neq 1 / 3$ with an appropriate choice of $c$. For example, we can set $\omega_{0}=1 / 5$ or $2 / 5$, and then choose

$$
c=\frac{1}{6} \sqrt{10 \pm 2 \sqrt{5}} .
$$

When $\tau_{0}=0$ at a fifth order resonance, Th. 5 in the Appendix implies that the fixed point is unstable at the bifurcation point. The corresponding map with $\omega \approx 1 / 5$ is shown in Fig. 9. The instability is difficult to see numerically because the size of the nonlinear terms is so small near the fixed point, though in the figure it is clear that the unstable motion does approach the origin. It is interesting to note that the instability is much more pronounced for $\omega=1 / 5$ than for $\omega=2 / 5$.

The second twist $\tau_{1}$ can be used in two different ways. For the cubic map with $d=0$ we can show that if $\tau_{0}=0$ then always $\tau_{1} \neq 0$, so that the only exceptions to stability can be the third through sixth order resonances. The value of $c$ that makes $\tau_{0}=0$ at $t=t_{0}$ and the corresponding value of $\tau_{1}$ are

$$
c=\frac{5-3 t_{0}^{2}}{3 t_{0}\left(t_{0}^{2}-3\right)}, \quad \pi \tau_{1}=\frac{105-305 t_{0}^{2}+353 t_{0}^{4}-167 t_{0}^{6}+30 t_{0}^{8}}{48 t_{0}^{3}\left(t_{0}^{2}-3\right)^{3}\left(t_{0}^{2}-1\right)} .
$$

It is easy to see that the polynomial in the numerator of $\tau_{1}$ is positive, so that $\tau_{1}$ is always nonzero if $\tau_{0}$ vanishes for the cubic map.

For the quartic map with $d \neq 0$ we can make both twists vanish at arbitrary $\omega_{0}$, in particular at seventh order resonance. In general the twists 


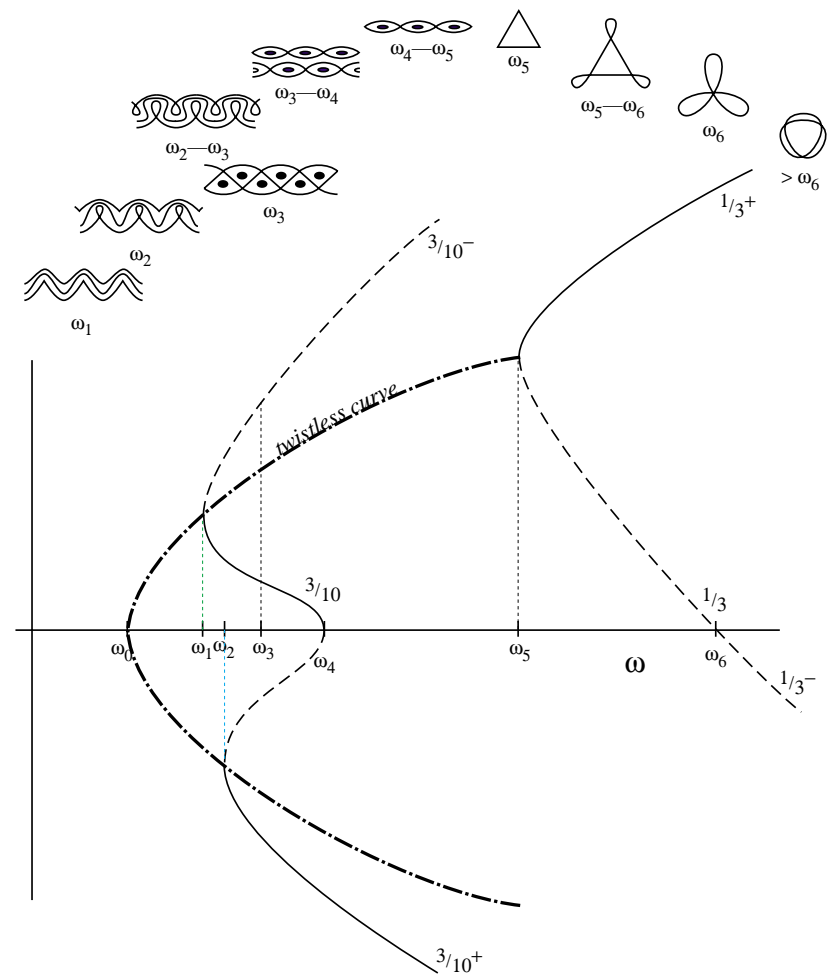

Figure 8: Sketch of the 3/10 and 1/3 bifurcations for the Hénon map as a function of $\omega$. Representative phase portraits are also shown. See Table 1 for bifurcation values.

vanish when

$$
c=\frac{5-3 t_{0}^{2}}{3 t_{0}\left(t_{0}^{2}-3\right)}, \quad d=\frac{105-305 t_{0}^{2}+353 t_{0}^{4}-167 t_{0}^{6}+30 t_{0}^{8}}{9 t_{0}^{2}\left(t_{0}^{2}-3\right)^{2}\left(t_{0}^{2}-1\right)\left(5 t^{2}-7\right)} .
$$

Thus if we choose $t_{0}=\tan (\pi / 7)$ we obtain $c \approx 1.0763012774$ and $d \approx$ .7144291292 . By Th. 5 the fixed point is unstable for these parameter values. Note that if we let $c$ and $d$ be the above functions of $\omega$ we obtain the very special one parameter family of quartic maps for which the first two twists vanish for every frequency. 

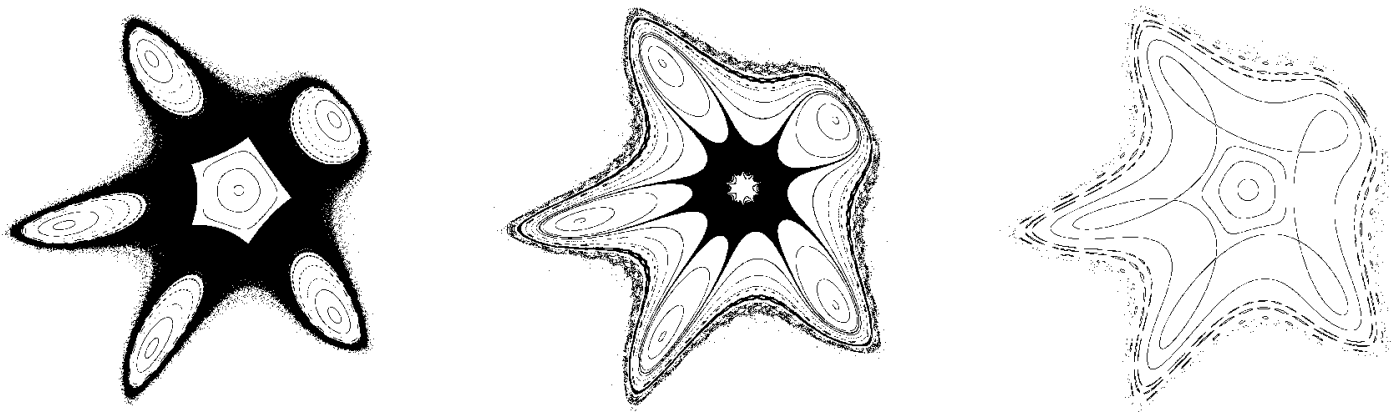

Figure 9: Phase portrait of the cubic map $(d=0)$ with $\tau_{0}=0$ at $\omega=1 / 5-$ $\epsilon, 1 / 5,1 / 5+\epsilon, \epsilon=0.0004$. At $\omega=1 / 5$ the fixed point is shown to be unstable.

\section{Conclusion}

By analyzing the normal form for Hamiltonian flows and area-preserving maps in the neighborhood of the tripling bifurcation we have shown that there exists a bifurcation creating a twistless torus. Thus in the presence of a tripling bifurcation, the non-degeneracy condition of the KAM theorem is violated on a one parameter family of tori in phase space in the neighborhood of the bifurcation. Moreover, in the nonintegrable case, reconnection bifurcations occur for orbits that collide at the twistless torus, such as the 3/10 orbits of the Hénon map in Fig. 8.

Using the notation of Fig. 8, we say that a twistless bifurcation occurs at $\omega=\omega_{0}$. The pair of period three orbits is created in a saddle-center bifurcation at $\omega_{5}$, and the tripling occurs at $\omega_{6}=1 / 3$. For the case of the resonant Hamiltonian normal form, Eq. (14) implies that the relative positions of these bifurcations have a simple ratio

$$
\frac{\omega_{0}-1 / 3}{\omega_{5}-1 / 3}=\frac{8}{3}
$$

Note that this ratio for the Hénon map is 2.8069 , only a $5 \%$ error. Since the Hamiltonian resonant normal form is integrable, we can follow the twistless torus; it exists when the rotation number of the central periodic orbit is in the interval $\omega_{0}$ to $\omega_{5}$. Moreover, the rotation number of the twistless curve itself, $\Omega_{0}$, takes all of the values in the interval from $\omega_{0}$ to $1 / 3$. For every rotation number in this interval there will generically be a reconnection bifurcation for 
the full two degree of freedom Hamiltonian. Hence this type of bifurcation occurs generically in nonintegrable Hamiltonian systems.

Similarly any area-preserving map that has a tripling bifurcation of an elliptic fixed point and that satisfies the nondegeneracy condition that the coefficient, $a_{02}$, does not vanish, has a twistless bifurcation. The dependence of the twist on rotation number is more complicated for this case than for the Hamiltonian, nevertheless, the rotation number of the fixed point unfolds the bifurcation. Moreover, there is always an $\omega \in[1 / 6,1 / 2)$ for which the resonant normal form has a twistless bifurcation.

As an example, the Hénon map has a twistless bifurcation at $k=9 / 16$, and the twistless curve moves outward as $k$ increases, causing reconnection bifurcations. We examined in particular the bifurcations of the 3/10 orbits. The vanishing of twist(s), as we showed for a cubic/quartic areapreserving map, can also lead to the instability of the fixed point at quintupling/septupling bifurcations.

The whole scenario is similar for the quadrupling bifurcation, when the resonant term dominates the twist term in magnitude (see the Appendix). In this case a pair of period 4 orbits are created in a saddle-center bifurcation. and a twistless torus is created at a cusp in the bifurcation diagram similar to that in Fig. 1. However, in this case the twistless curve does not collide with the fixed point, and a simple prediction about what rotation numbers must pass through the twistless curve is not possible.

It would be interesting to generalize these results to higher dimensional systems.

\section{Appendix: Instability of resonant maps without twist}

In this section we give sufficient conditions for which a map of the plane with eigenvalues that are $n^{\text {th }}$ roots of unity has an unstable fixed point. Here the condition of area preservation is not necessary. Examples of this behavior are well known [13], and necessary and sufficient conditions for instability have been determined in $[14,1]$. However, we have not found a simple and explicit proof of instability for the case where the resonant term has the lowest possible order.

To study this problem, we put the map in the normal form Eq. (7). We 
will show that the fixed point is generically unstable whenever all of the twist terms vanish that are of lower order than $O(n-1)$, the order of the resonant term. When $n$ is even, there is a twist term of the same order as the resonant term. In this case the $O(n-1)$ twist term need not vanish, but its magnitude must be dominated by the resonant term.

Theorem 5. Let $f$ be a $C^{n}$ map of the plane with a fixed point at the origin. Suppose that the multipliers of the fixed point are $n^{\text {th }}$ roots of unity, and that when $f$ is put in normal form, the low order twist coefficients vanish:

$$
a_{j, j-1}=0 \quad, \quad 1<j<\frac{n}{2},
$$

but the lowest order resonant term is nonzero

$$
a_{0, n-1} \neq 0 .
$$

If $n$ is even, assume in addition that

$$
\left|a_{n / 2, n / 2-1}\right|<\left|a_{0, n-1}\right| .
$$

Then the origin is unstable.

This instability is well known. Our goal in this appendix is to give a simple, explicit proof.

Note that when $n=3$, the assumed form is generic, but for $n=4$, the first twist, $a_{21}$, is assumed to be small, and when $n=5$ it is assumed to vanish. As we saw in $\S 3.2$ the first two twists vanishes generically in a two parameter area-preserving family; one parameter to adjust the frequency and the second one to make the first twist vanish.

Proof. When $n$ is odd the assumed normal form is

$$
z^{\prime}=\lambda\left(z+\alpha \bar{z}^{n-1}\right)+O(n) .
$$

We adapt the proof from Siegel and Moser [13]. Begin by scaling $z$ using the transformation $z \rightarrow z s$ where $s$ determined by $\bar{s}^{n-1} / s=(n \alpha)^{-1}$. Then, using the fact that $\lambda^{n}=1$, the $n^{\text {th }}$ power of $z^{\prime}$ becomes

$$
\left(z^{\prime}\right)^{n}=z^{n}+z^{n-1} \bar{z}^{n-1}+O(2 n-1) .
$$

Introducing new coordinates $Z$ by

$$
z^{n}=Z=X+i Y=R \exp i \Phi,
$$


gives

$$
Z^{\prime}=Z+R^{2-2 / n}\left(1+\eta(Z, \bar{Z}) R^{1 / n}\right),
$$

where the higher order terms have been represented by a factor $\eta$, which, since $f$ is $C^{n}$, is bounded as $R \rightarrow 0$. The purpose of the scaling to eliminate $\alpha$ is that the real part of this map now becomes

$$
X^{\prime}=X+R^{2-2 / n}\left(1+\Re(\eta) R^{1 / n}\right) .
$$

Since $\eta$ is bounded, there is an $R_{0}$ such that for all $R<R_{0}$, we have $\left|\Re(\eta) R^{1 / n}\right|<1 / 2$. Thus whenever $R<R_{0}, X^{\prime}>X+\frac{1}{2} R^{2-2 / n}$, and so $X$ is monotone increasing in each step of the map. It is easy to see that this implies that the fixed point is unstable.

The case of even $n$ is slightly harder because there can be a twist term of the same order:

$$
z^{\prime}=\lambda\left(z+\alpha \bar{z}^{n-1}+\beta z|z|^{(n-2)}+O(2 n-1)\right) .
$$

If $\beta$ happens to be zero the above proof works. When $\beta$ is nonzero the stability of the fixed point is determined by the relative size of the resonant term and the twist term. Again we scale away $\alpha$ and the $n^{\text {th }}$ power of the new map is

$$
\left(z^{\prime}\right)^{n}=z^{n}+(z \bar{z})^{n-1}+\tilde{\beta} z^{n}|z|^{(n-2)}+O(2 n-1),
$$

where $\tilde{\beta}=\beta /|\alpha|$. In the new coordinates this becomes

$$
Z^{\prime}=Z+R^{2-2 / n}\left(1+\tilde{\beta} \exp (i \Phi)+\eta R^{1 / n}\right),
$$

and the map for the real part is

$$
X^{\prime}=X+R^{2-2 / n}\left(1+\Re(\tilde{\beta} \exp (i \Phi))+\Re(\eta) R^{1 / n}\right) .
$$

So if $1+\Re(\tilde{\beta} \exp (i \Phi))>0$ for all $\Phi$ the argument of the previous case applies. Therefore the instability criterion is $|\beta| /|\alpha|<1$ : if the "twist" term is smaller than the resonant term the mapping is unstable. The well known example of this type is the quadrupling bifurcation. Our calculation shows that a similar codimension two bifurcation occurs for the area-preserving sextupling bifurcation. 


\section{References}

[1] D. Aharonov and U. Elias. Parabolic fixed points, invariant curves and action-angle variables. Ergod. Th. Dynam. Sys., 10:231-245, 1990.

[2] V.I. Arnold. Mathematical Methods of Classical Mechanics. Springer, New York, 1978.

[3] A.M. Ozorio de Almeida. Hamiltonian Systems: Chaos and Quantization. Cambridge University Press, Cambridge, 1990.

[4] D. del Castillo-Negrette, J.M. Greene, and P.J. Morrison. Area preserving nontwist maps: Periodic orbits and transition to chaos. Physica D, 91(1):1-23, 1996.

[5] A. Delshams and R. de la Llave. KAM theory and a partial justification of Greene's criterion for non-twist maps. preprint 98-732 mp_arc@math.utexas.edu, 1998.

[6] M. Hénon. Numerical study of quadratic area-preserving mappings. Quart. Appl. Math., 27:291-312, 1969.

[7] J. E. Howard and J. Humpherys. Nonmonotonic twist maps. Physica D, 80(3):256-276, 1995.

[8] J.E. Howard and S.M. Hohs. Stochasticity and reconnection in hamiltonian systems. Physical Review A, 29:418, 1984.

[9] J.D. Meiss. Symplectic maps, variational principles, and transport. Reviews of Modern Physics, 64(3):795-848, 1992.

[10] K.R. Meyer and G.R. Hall. Introduction to the Theory of Hamiltonian Systems, volume 90 of Applied Mathematical Sciences. Springer-Verlag, New York, 1992.

[11] J.K. Moser. On quadratic symplectic mappings. Math. Zeitschrift, 216:417-430, 1994.

[12] H. Schomerus. Periodic orbits near bifurcations of codimension two: classical mechanics, semiclassics and Stokes transitions. J. Phys. A, 31(18):4167-4196, 1998. 
[13] C.L. Siegel and J.K. Moser. Lectures on Celestial Mechanics. Classics in Mathematics. Springer-Verlag, New York, 1971.

[14] C. Simó. Stability of degenerate fixed points of analytic area preserving mappings. Astérisque, 98-99:184-194, 1982.

[15] C. Simó. Invariant curves of analytic perturbed nontwist area preserving maps. Regular $\& 3$ Chaotic Dynamics, 3:180-195, 1998.

[16] C. Simó and D. Treschev. Evolution of the "last" invariant curve in a family of area preserving maps. preprint, www.maia.ub.es, 1998.

[17] D. Sterling, H.R. Dullin, and J.D. Meiss. Homoclinic bifurcations for the Hénon map. Physica D, 134:153-184, 1999.

[18] J. P. Van der Weele and T. P. Valkering. The birth process of periodic orbits in nontwist maps. Physica A, 169(1):42-72, 1990. 